Eloisio do Carmo Lourenço ${ }^{1}$

Luciane Miranda Guerra

Rogerio Antonio Tuon ${ }^{1}$

Sandra Maria Cunha Vidal e Silva ${ }^{1}$

Glaucia Maria Bovi Ambrosano ${ }^{1}$

José Eduardo Corrente ${ }^{2}$

Karine Laura Cortellazzi ${ }^{1}$

Fabiana de Lima Vazquez ${ }^{1}$

Marcelo de Castro Meneghim ${ }^{1}$

Antonio Carlos Pereira ${ }^{1}$

\section{Variáveis de impacto na queda da mortalidade infantil no Estado de São Paulo, Brasil, no período de 1998 a 2008}

Impact variables on the decline in infant mortality

in the state of São Paulo, Brazil: 1998-2008
Abstract This is an ecological, analytical and retrospective study comprising the 645 municipalities in the State of São Paulo, the scope of which was to determine the relationship between socioeconomic, demographic variables and the model of care in relation to infant mortality rates in the period from 1998 to 2008. The ratio of average annual change for each indicator per stratum coverage was calculated. Infant mortality was analyzed according to the model for repeated measures over time, adjusted for the following correction variables: the city's population, proportion of Family Health Programs (PSFs) deployed, proportion of Growth Acceleration Programs (PACs) deployed, per capita GDP and SPSRI (São Paulo social responsibility index). The analysis was performed by generalized linear models, considering the gamma distribution. Multiple comparisons were performed with the likelihood ratio with chi-square approximate distribution, considering a significance level of 5\%. There was a decrease in infant mortality over the years ( $p<$ 0.05), with no significant difference from 2004 to 2008 ( $p>0.05)$. The proportion of PSFs deployed $(p<0.0001)$ and per capita GDP $(p<0.0001)$ were significant in the model. The decline of in fant mortality in this period was influenced by the growth of per capita GDP and PSFs.

Key words Infant mortality, Socioeconomic factors, National strategies
Resumo Trata-se de estudo ecológico analítico, retrospectivo, composto pelos 645 municípios do Estado de São Paulo, cujo objetivo foi verificar a relação entre variáveis socioeconômicas, demográficas e modelo de atenção, em relação ao coeficiente de mortalidade infantil, no período de 1998 a 2008. Foi calculada a proporção de variação média anual para cada indicador por estrato de cobertura. A mortalidade infantil foi analisada segundo modelo de medidas repetidas no tempo, ajustado para as variáveis de correção: população do município, proporção de PSF implantado, proporção de PACS implantado, PIB per capita e IPRS (indice paulista de responsabilidade social). A análise foi realizada por modelos lineares generalizados, considerando a distribuição gama. Comparações múltiplas foram realizadas pela razão de verossimilhança com distribuição aproximada quiquadrado, considerando-se nivel de significância de 5\%. Houve diminuição da mortalidade infantil no decorrer dos anos ( $p<0,05)$, não havendo diferença significativa de 2004 a 2008 ( $p>0,05)$. A proporção de PSF implantado $(p<0,0001)$ e o PIB per capita $(p<0,0001)$ foram significativos no modelo. A queda da mortalidade infantil no período analisado foi influenciada pelo crescimento do PIB per capita e pelo modelo Saúde da Família. Palavras-chave Mortalidade infantil, Fatores socioeconômicos, Estratégias Nacionais
Brasil.

eloisio@matrix.com.br

${ }^{2}$ Instituto de Biociência,

UNESP Campus Botucatu. 


\section{Introdução}

A taxa de mortalidade infantil é um indicador que expressa a combinação de fatores individuais da mãe da criança, contextuais, hereditários e relacionados à atenção à saúde para crianças menores de um ano. É, portanto, um indicador da condição de vida e saúde da população ${ }^{1}$.

O empenho do Brasil para a redução da mortalidade infantil tem sido observado através dos compromissos internos e externos, firmados para a melhoria da qualidade da atenção à saúde prestada à gestante e ao recém-nascido. Tanto assim, que no ano de 2004, no âmbito da Presidência da República, foi firmado o "Pacto pela Redução da Mortalidade Materna e Neonatal" com o objetivo de articular os atores sociais mobilizados em torno da melhoria da qualidade de vida de mulheres e crianças ${ }^{1}$.

No cenário internacional, o Brasil assumiu a luta pela redução da mortalidade infantil, como parte das Metas do Desenvolvimento do Milênio.

Esse compromisso, que visa o alcance de patamares mais dignos de vida para a população, foi assumido pelos países integrantes da Organização das Nações Unidas (ONU) e ainda desafia os serviços de saúde e a sociedade como um todo ${ }^{2}$.

O resultado de tais esforços se reflete em expressiva queda da mortalidade infantil no país ${ }^{3}$.

Vários estudos atuais têm relacionado os níveis de renda com a mortalidade infantil ${ }^{3-5}$. Embora a taxa de mortalidade infantil venha caindo, mesmo em países não desenvolvidos, estudos têm mostrado uma relação positiva entre renda e saúde desde a primeira infância ${ }^{6}$. Pessoas mais pobres geralmente vivem em piores condições ambientais e, portanto, estão mais expostas a doenças. Da mesma forma, têm menor acesso a serviços de saúde de boa qualidade ${ }^{7}$. Estudo americano de Case et al. ${ }^{8}$, em 2002, mostrou que a renda domiciliar influencia positivamente nas condições de saúde de crianças com idade entre 0 e 17 anos, além de apontar ainda para a relação com a escolaridade dos pais.

No Brasil, a taxa de mortalidade infantil teve redução de 50,8\% entre os anos 2000 e 2010, segundo a Secretaria de Avaliação e Gestão de Informação do Ministério do Desenvolvimento Social e de 47,5\% segundo o IBGE ${ }^{3}$.

Análise mais detalhada dessa queda aponta que os coeficientes de mortalidade infantil foram substancialmente reduzidos, com taxa anual de decréscimo de 5,5\% nas décadas de 1980 e 1990 e $4,4 \%$ no período $2000-08$, atingindo vinte falecimentos por 1.000 nascidos vivos em 2008, sendo que os de neonatais foram responsáveis por $68 \%$ das mortes infantis ${ }^{9}$. O cuidado adequado ao recém-nascido tem sido, portanto, um dos desafios para reduzir esses índices. Contudo, observa-se menor velocidade no componente neonatal precoce (0-6 dias de vida $)^{1}$.

Estudos conduzidos por Barros et al. ${ }^{10}$, em 2010, evidenciam que medidas pró-ativas para reduzir as disparidades de saúde, acompanhados pelo progresso socioeconômico podem resultar em melhorias mensuráveis na saúde das crianças e das mães em um intervalo relativamente curto. Isso demonstra o sucesso do Brasil diante do desafio assumido em alcançar as metas de desenvolvimento do milênio, especificamente a meta 4, que é a de redução da mortalidade infantil.

A Estratégia de Saúde da Família, dêsde sua implantação em 1994, tem se baseado no paradigma da ampliação das ações de prevenção e promoção em saúde, buscando a substituição de práticas, ainda preponderantes, fundamentadas na formação superespecializada, com dependência dos recursos tecnológicos complexos e um isolamento entre as disciplinas de saúde ${ }^{11,12}$.

Considerada também como a principal estratégia do SUS para avançar na conquista da integralidade e equidade na Atenção Básica, a Estratégia da Saúde da Família propõe melhorar e facilitar o acesso das pessoas aos serviços de saúde, melhorar o vínculo de confiança, conhecer as particularidades do território, já que cada equipe se responsabiliza pelo acompanhamento de 600 a 1000 famílias (cerca de 3.500 pessoas) de uma área adstrita ${ }^{13}$. Busca-se, assim, a melhoria dos indicadores locais de saúde no território e, consequentemente, de todo o país, conforme o aumento da cobertura da ESF.

Pesquisa realizada em parceria entre a Secretaria de Vigilância em Saúde (Ministério da Saúde) e a Universidade de Nova York, concluiu que o modelo de atenção à saúde, no caso da Estratégia de Saúde da Família, foi um fator de maior efeito na queda da mortalidade infantil, no Brasil, entre 1990 e 2002, do que o acesso a hospitais, o aumento do número de médicos ou a ampliação do acesso à água tratada ${ }^{14}$.

Todavia, há que se se obter evidências factuais que sustentem a expansão da saúde da família nos grandes centros urbanos do país a fim de se legitimar sua consolidação, já que, segundo Fachini et al. ${ }^{15}$, em 2006, o adequado enfrentamento dos principais problemas da estratégia poderá incrementar significativamente seu desempenho e impactar positivamente na saúde da população. 
Nesse sentido, a Estratégia de Saúde da família, necessita passar por processos avaliativos criteriosos que estabeleçam suas relações com as mudanças de indicadores observadas após sua implantação, o que aponta para a imprescindibilidade dos estudos que se propõem discutir a associação entre a ESF e a queda da mortalidade infantil de uma maneira mais conjuntural. Muitos eventos ocorrem ao mesmo tempo, tais como a melhora dos indicadores da economia ou do contexto em que a pessoa vive, os quais também podem contribuir com tais mudanças.

Quando são realizadas múltiplas análises, a veracidade da correlação entre as mudanças dos indicadores com a cobertura da ESF é mais profundamente analisada, o que poderia ser recomendável na avaliação de políticas e programas de saúde.

O objetivo deste estudo foi verificar a relação de variáveis socioeconômicas, demográficas e do modelo de atenção em relação ao coeficiente de mortalidade infantil, no estado de São Paulo, no período de 1998 a 2008.

\section{Métodos}

Este trabalho foi submetido à aprovação ao Comitê de Ética em Pesquisa (CEP) da FOP - UNICAMP, conforme resolução 196/96 ${ }^{16}$, de 10/10/ 1996 do Conselho Nacional de Saúde do Ministério da Saúde. Como os dados utilizados são secundários, provenientes de bases de dados públicos, foi dispensado de apreciação pelo CEP.

Trata-se de estudo ecológico analítico, retrospectivo composto por todos os 645 municípios do Estado de São Paulo. Desse total, 468 apresentavam Equipes do Programa Saúde da Família até dezembro de 2008, segundo dados do $\mathrm{MS}^{17}$.

Os dados referentes ao número de Equipes de Saúde da Família (ESF), bem como os indicadores da atenção básica foram obtidos do Sistema de Informação da Atenção Básica (SIAB), disponíveis no site do Departamento de Atenção Básica do Ministério da Saúde (MS) ${ }^{18}$.

Os dados referentes ao IPRS (Índice paulista de responsabilidade social) foram coletados através do site da Fundação Seade (Sistema Estadual de Análise de Dados Estatísticos) do governo do estado de São Paulo. Esse índice tem como finalidade caracterizar os municípios paulistas no que se refere ao desenvolvimento humano, por meio de indicadores sensíveis a variações de curto prazo e capazes de incorporar informações referentes às diversas dimensões que compõem o índi- ce. Nesse sentido, ele preserva as três dimensões consagradas pelo IDH - riqueza, longevidade e escolaridade. O IPRS é classificado em grupo 1, 2, 3, 4 e 5; sendo o grupo 1 a melhor situação e o 5 a pior. No ranking da dimensão "riqueza" do índice os municípios do Estado são classificados de 1 a 645 .

Em relação ao PIB, os dados foram obtidos através do IBGE (Instituto Brasileiro de Geografia e Estatística).

Os dados populacionais foram obtidos do Datasus e das projeções calculadas pelo IBGE.

Todos os dados analisados são secundários provenientes de bancos de dados públicos. Não houve participação de indivíduos na pesquisa.

Os dados sobre a estimativa da proporção da população coberta pelo PSF foram obtidos através do critério de uma equipe para cada 3.450 pessoas (cálculo padrão adotado pelo Departamento de Atenção Básica do Ministério da Saúde). O mês, tomado como referência para a informação do número de Equipes da Saúde da Família, foi dezembro para cada ano.

O indicador de saúde de interesse para este estudo foi o coeficiente de mortalidade infantil, relacionado à saúde da criança, analisado de acordo com os estratos de cobertura do PSF; sendo que cada grupo de municípios dos estratos de cobertura teve sua situação de saúde analisada, retrospectivamente, para os anos de 1998 a 2008.

Foi calculada a proporção de variação média anual para cada indicador por estrato de cobertura.

\section{Análise Estatística}

Os resultados da evolução de implantação da ESF e as estimativas de população coberta por este programa para o Estado de São Paulo e seus municípios foram expressos em médias, frequências e proporções e para melhor compreensão através de gráficos e tabelas.

Para processamento, análise e apresentação gráfica dos dados foram utilizados os programas SAS e Excel.

A variável mortalidade infantil foi analisada no decorrer do tempo, segundo modelo de medidas repetidas no tempo, ajustado para as variáveis de correção (população do município, proporção de PSF implantado, proporção de PACS implantado, PIB per capita, IPRS e renda per capita). Como as variáveis não apresentaram distribuição normal, a análise foi realizada por modelos lineares generalizados, considerando a distribuição gama. As comparações múltiplas 
foram realizadas pela razão de verossimilhança com distribuição aproximada qui-quadrado, pela opção DIFF do GENMOD do programa SAS, considerando o nível de significância de 5\%.

\section{Resultados}

Entre as variáveis analisadas, apenas a proporção de PSF implantada $(\mathrm{p}<0,0001)$ e o PIB per capita ( $\mathrm{p}<0,0001)$ foram significativos no modelo de medidas repetidas no tempo, ou seja, influenciaram na variação da mortalidade no decorrer do período.

Assim, as comparações entre os anos foram realizadas, ajustando-se para essas variáveis. Houve diminuição da mortalidade infantil no decorrer dos anos $(\mathrm{p}<0,05)$, não havendo diferença significativa a partir de 2004 até 2008 (p > $0,05)$, conforme se pode observar na Tabela 1.

A tendência de queda da mortalidade infantil no período estudado foi acompanhada, no mesmo período, pelo aumento na cobertura pela Estratégia de Saúde da Família, como se observa no Gráfico 1.

Da mesma forma, observa-se que, à medida em que cresce o PIB, a mortalidade infantil apresenta queda no Estado de São Paulo de 1998 a 2008, como demonstra o Gráfico 2.

\section{Discussão}

A região Sudeste apresentou ampliação na cobertura populacional pela Estratégia de Saúde da Família de aproximadamente $24 \%$ no período de 1998 a 2008. Isso coloca a região num pata- mar de crescimento lento e gradual quando comparado a regiões menos favorecidas social e economicamente como, por exemplo, as regiões Norte e Nordeste, que apresentaram, no mesmo período, aumento na referida cobertura da ordem de $42,4 \%$ e $67 \%$, respectivamente ${ }^{15}$.

O modelo de atenção baseado na saúde da família privilegia as atividades de planejamento e desenvolvimento de ações intersetoriais, com priorização para a saúde da criança, acesso universal com escuta qualificada e encaminhamento para a resolução das demandas necessárias, responsabilização e vínculo entre os profissionais de saúde e usuários. Isso tudo, certamente impacta, tanto na ampliação do acesso, quanto na adesão do usuário ao tratamento, o que, consequentemente, qualifica o serviço, aumenta resolutividade e diminui, por fim, a mortalidade.

Isso ficou provado no presente estudo, ao se analisar os dados através de modelo multivariado, adotando matriz de covariâncias sem restrições, demonstrando uma relação forte $(\mathrm{p}<$ 0,0001) entre Estratégia de Saúde da Família e mortalidade, remetendo às melhorias no acesso aos serviços oferecidos no ESF, além das características e da organização do trabalho nessas equipes.

Ademais, considerando a lógica de integralidade da Estratégia, o atendimento na Saúde da Família não se restringe, via de regra, apenas às queixas apresentadas, mas à promoção da saúde com a compreensão de seus determinantes ambientais, interações familiares e com a comunidade, patologias prevalentes e aspectos culturais específicos daquela comunidade; sendo que a criança deve ser acompanhada pela atenção básica em toda a sua trajetória pelo Sistema. Com esse

\begin{tabular}{lcccccc}
\hline \multicolumn{6}{l}{ Tabela } \\
\hline 1. Mortalidade infantil no decorrer dos anos. \\
\hline Ano & Média & Desvio padrão & Valor mínimo & Valor máximo & IC (95\%) & Mediana \\
\hline 1998 & 18,94 & 14,01 & 0 & 83,33 & $17,85-20,01$ & 17,70 \\
1999 & $17,42^{\text {a }}$ & 14,37 & 0 & 125,00 & $16,31-18,53$ & 16,13 \\
2000 & $17,61^{\text {a }}$ & 13,59 & 0 & 90,91 & $16,55-18,66$ & 16,13 \\
2001 & $16,59^{\text {ab }}$ & 13,34 & 0 & 125,00 & $15,56-17,62$ & 15,71 \\
2002 & $15,45^{\text {bd }}$ & 13,53 & 0 & 111,11 & $14,40-16,49$ & 14,08 \\
2003 & $15,82^{\text {bc }}$ & 14,43 & 0 & 166,67 & $14,70-16,93$ & 14,20 \\
2004 & $14,54^{\text {cde }}$ & 12,74 & 0 & 100,00 & $13,55-15,52$ & 13,39 \\
2005 & $13,96^{\text {de }}$ & 13,21 & 0 & 117,65 & $12,93-4,97$ & 12,40 \\
2006 & $13,86^{\text {de }}$ & 12,98 & 0 & 86,96 & $12,85-14,86$ & 12,27 \\
2007 & $13,08^{\text {e }}$ & 12,72 & 0 & 107,14 & $12,09-14,06$ & 11,83 \\
2008 & $13,36^{\text {e }}$ & 13,01 & 0 & 111,11 & $12,35-14,36$ & 12,08
\end{tabular}

*Análise ajustada para as variáveis de correção. Médias seguidas de letras distintas diferem entre $s i(p \leq 0,05)$. 


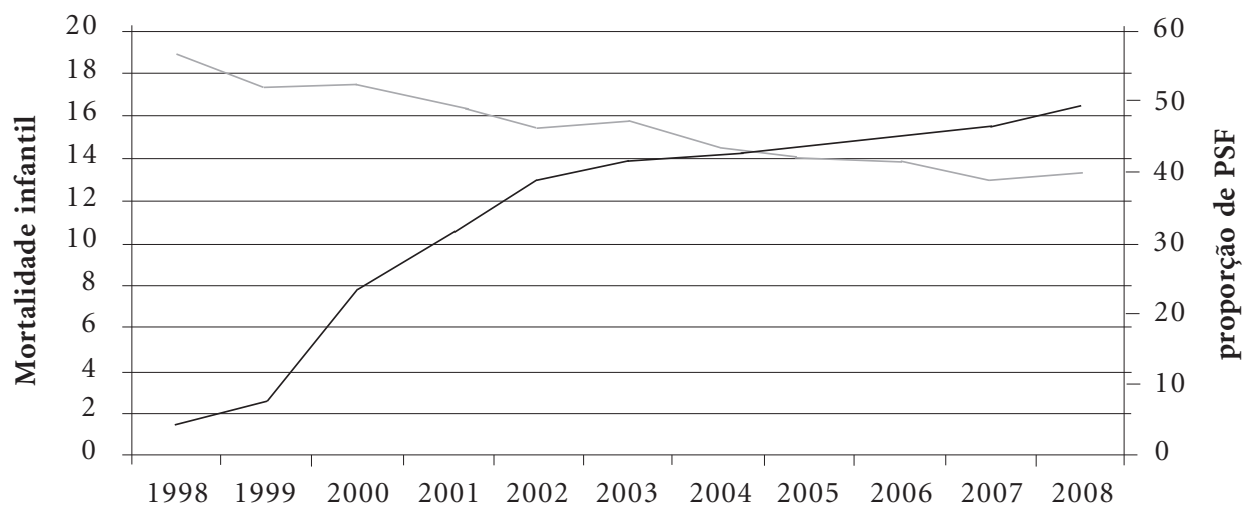

Ano

Gráfico 1. Mortalidade infantil e proporção de PSF no decorrer do tempo.

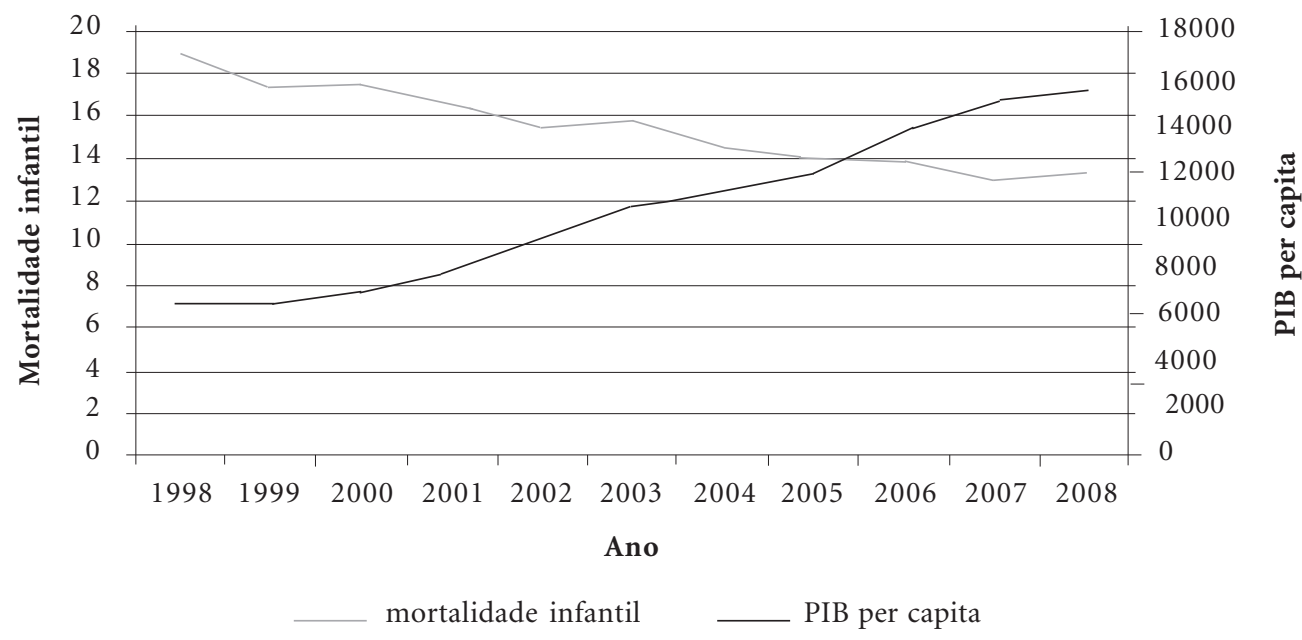

Gráfico 2. Mortalidade infantil e o PIB per capita no decorrer do tempo.

enfoque global e integral, fica clara a importância dessa estratégia num cenário onde se sabe que tais determinantes interferem na mortalidade infantil.

O presente estudo corrobora os resultados encontrados por Macinko et al. ${ }^{19}$, em estudo ecológico incluindo 537 microrregiões no Brasil, no qual depois de controlarem outros determinantes da saúde, observaram que um aumento de cobertura de PSF em 10\% acarretava uma queda de $0,45 \%$ na taxa de mortalidade infantil, sendo de $0,6 \%$ no componente pós-neonatal e de $1 \%$ na mortalidade por diarreia. Além disso, em nível estadual, confirma, com a devida ponderação, as conclusões de Lira et al. ${ }^{20}$, que apontaram para a maior ocorrência dos óbitos evitáveis em menores de 1 ano em áreas não cobertas pelo programa Saúde da Família. 
O processo histórico e político de implantação do PSF dentro do Estado de São Paulo foi muito mais lento que em outras regiões do país nesse período, o que pode tornar os dados ainda mais interessantes e contumazes em relação ao efetivo impacto das ações.

Cabe ressaltar, contudo, conforme apontam Costa et al. ${ }^{21}$, que as análises e a interpretação de dados secundários, no Brasil, requerem critério, já que os Sistemas de Informação apresentam diferentes estágios de desenvolvimento e uniformidade.

O presente estudo demonstra que a interferência do modelo de atenção na queda da mortalidade se deu de 1994 a 2004; diminuindo tal efeito, contudo, no período de 2004 a 2008.

Isso nos faz refletir sobre as premissas que norteiam modelos de saúde. Trata-se da necessidade de se rever processos de trabalho que inicialmente se mostram eficazes, mas que podem esgotar-se; métodos gerenciais que - face aos desafios da modernidade - necessitam de revisão ao longo do tempo.

Nesse sentido, o presente estudo vem acrescentar importantes fundamentos ao tema, na medida em que avalia a mortalidade ao longo do tempo, levando em conta, para isso, a interferência dos fatores citados, sendo possível, assim, compreender a significância de cada fator em função do tempo.

Ao apontar a interferência de fatores socioeconômicos com a queda da mortalidade infantil, corrobora estudo de Soares e Menezes ${ }^{22}$, em 2010, que demonstraram a importância da variável escolaridade no enfrentamento da mortalidade infantil, bem como os de Szwarcwald et al. ${ }^{23}$, que apontaram a relação da mortalidade infantil com problemas econômicos e dificuldades de acesso aos serviços de saúde.
O cuidado em analisar variáveis de contexto conjuntamente com as de modelo de atenção, através de modelos estatísticos apropriados é relevante nos estudos citados, pois minimiza possíveis equívocos de interpretação dos resultados, bem como evita conclusões redundantes.

Esses dados corroboram conclusões de estudo anterior, realizado na Bahia, o qual demonstrou associação entre aumento da mortalidade infantil e o período de recessão mais acentuada, expressa na queda dos PIB nacional e daquele Estado $^{24}$. Assim, ao demonstrar a queda expressiva - de aproximadamente $33 \%$ - no coeficiente de mortalidade infantil no Estado de São Paulo, o presente estudo confirma a necessidade de se considerar, na análise das tendências de mortalidade infantil, a força das variáveis socioeconômicas e não somente os modelos de atenção à saúde.

Contudo, trata-se de um estudo ecológico, cuja inferência requer cuidados, sendo que sua continuidade, através de novas pesquisas, revela-se oportuna, adequada e indicada no momento atual, em que se observa crescimento econômico do país, além de acentuada velocidade de implantação da Estratégia de Saúde da Família.

\section{Conclusão}

Durante o período estudado, a queda da mortalidade infantil observada no Estado de Estado de São Paulo foi influenciada pelo crescimento do PIB per capita e pelo modelo de atenção Saúde da Família, sendo que o impacto do modelo de atenção não foi significante após 2003.

A análise da não continuidade do impacto do modelo de atenção em tal queda após 2003 requer novos estudos, a fim de que se possa testar possíveis fatores intervenientes. 


\section{Colaboradores}

EC Lourenço foi responsável pela concepção e o delineamento do estudo, bem como pela redação do artigo; LM Guerra contribuiu na discussão dos resultados, conclusão e na revisão literária do assunto para o artigo; RA Tuon e SMCV Silva contribuiram na discussão dos dados do artigo; GMB Ambrosano e José Eduardo Corrente realizaram a análise estatística e interpretação dos resultados da pesquisa; KL Cortellazzi, FL Vazquez e MC Meneghim contribuíram na discussão dos resultados da pesquisa; AC Pereira orientou a pesquisa, a elaboração do artigo e contribuiu na interpretação e discussão dos resultados.

\section{Referências}

1. Brasil. Ministério da Saúde (MS). Atenção à Saúde do Recém-Nascido. Guia para os profissionais de saúde. Cuidados gerais. Brasília: MS; 2011. Vol. 1.

2. Brasil. Ministério da Saúde. Secretaria de Vigilância em Saúde. Departamento de Análise de Situação de Saúde. Coordenação Geral de Informação e Análise Epidemiológica. Manual de vigilância do óbito infantil e fetal e do comitê de prevenção do óbito infantil e fetal. Brasília: MS; 2009.

3. Brasil. Ministério do Desenvolvimento e Combate à Fome (MDS). Secretaria de avaliação e gestão da informação. Taxas de mortalidade infantil por região e faixa de renda domiciliar per capita entre os censos de 2000 e 2010. Brasília: MDS; 2012. Estudo técnico N. ${ }^{\circ}$ 05/2012.

4. Determinantes Sociais da Saúde (DSS). Portal e observatório sobre iniqüidades em saúde. Todos pela equidade. Biblioteca Virtual em Saúde (BVS). [acessado 2013 maio 8]. Disponível em: http:// dssbr.org/site/2012/04/tendencias-de-mortalidadeinfantil-e-na-infancia-no-brasil/

5. Wilkinson R. Saúde, economia e sociedade. Relação internacional entre eqüidade de renda e expectativa de vida. In: Barata RB, Barreto ML, Almeida Filho N, Veras RP, organizadores. Equidade e saúde: contribuições da epidemiologia. Rio de Janeiro: Fiocruz; 1997.

6. Deaton A. What does the empirical evidence tell us about the injustice of health inequalities? In: Nordheim OF, Hurst SA, Norheim OF, Wiclker D, editors. Inequalities in health: ethics and measurement. Oxford: Oxford University Press; 2012.

7. Geib LTC, Fréu CM, Brandão M, Nunes ML. Determinantes sociais e biológicos da mortalidade infantil em coorte de base populacional em Passo Fundo, Rio Grande do Sul. Cien Saude Colet 2010; 15(2):363-370.

8. Case A, Lubotsky D, Paxson C. Economic Status and Health in Childhood: The Origins of the Gradient. The American Economic Review 2002; 92(5): 1308-1334.

9. Victora CG, Aquino EML, Leal MC, Monteiro CA Barros FC, Szwarcwald CL. Saúde de mães e crianças no Brasil: progressos e desafios. Lancet 2011; $32: 46$.

10. Barros FC, Matijasevich A, Requejo JH, Giugliani E, Maranhão AG, Monteiro CA, Barros AJD, Bustreo F, Merialdi M, Victora CG. Recent trends in maternal, newborn, and child health in Brazil: progress toward Millennium development goals 4 and 5. Am J Public Health 2010; 100(10):1877-1889.

11. Ronzani TM, Van Stralen CJ. Dificuldades de Implantação do Programa de Saúde da Família como Estratégia de Reforma do Sistema de Saúde Brasileiro. Revista APS 2003; 6(2):99-107.

12. Mattos GCM, Leite ICG, Greco R M. A inclusão da equipe de saúde bucal na Estratégia Saúde da Família: entraves, avanços e desafios. Cien Saude Colet 2014; 19(2):373-382. 
13. Brasil. Ministério da Saúde (MS). Portaria no 2.488, de 21 de outubro de 2011. Aprova a Política Nacional de Atenção Básica, estabelecendo a revisão de diretrizes e normas para a organização da Atenção Básica, para a Estratégia Saúde da Família (ESF) e o Programa de Agentes Comunitários de Saúde (PACS). Diário Oficial da União 2011; 22 out.

14. Brasil. Ministério da Saúde (MS). Sistema Único de Saúde. Painel de Indicadores do SUS no 4. Temático Saúde da Família v II. Brasília: MS; 2008.

15. Facchinni LA, Piccini RX, Tomasi E, Thumé E, Silveira DS, Siqueira FV, Rodrigues MA. Desempenho do PSF no Sul e no Nordeste do Brasil: avaliação institucional e epidemiológica da Atenção Básica à Saúde. Cien Saude Colet 2006; 11(3):669-681.

16. Brasil. Ministério da Saúde (MS). Conselho Nacional de Saúde. Resolução no. 196 de 10 de outubro de 1996. Diretrizes e Normas Regulamentadoras de Pesquisas Envolvendo Seres Humanos. Diário Oficial da União 1996; 16 out.

17. Brasil. Ministério da Saúde (MS). Secretaria de Atenção à Saúde. Departamento de Atenção Básica. Saúde da Família. [acessado 2013 fev 28]. Disponível em: http://dab.saude.gov.br/atencaobasica.php\#saudeda familia

18. Brasil. Ministério da Saúde (MS). Secretaria de Atenção à Saúde. Departamento de atenção básica. Saúde da Família. Números da Saúde da Família. [acessado 2013 maio 10]. Disponível em: http://189.28. 128.100/dab/docs/abnumeros/historico_1998.pdf

19. Macinko J, Guanais FC, Souza, MFM. An evaluation of impact of the Family Health Program on infant mortality in Brazil, 1990-2002. J Epidemiol Community Health 2006; 60(1):13-19.

20. Lira KP, Ratis CAS, Farias MMC, Gomes SF, Cortez MR. PSF como estratégia de controle dos óbitos infantis por causas evitáveis no Recife (2000 a 2003). In: VI Congresso Brasileiro de Epidemiologia. Livro de Resumos [CD-ROM]. Olinda: Abrasco; 2004.

21. Costa MCN, Mota ELA, Paim JS, Silva LMV, Teixeira MG, Mendes CMC. Mortalidade infantil no Brasil em períodos recentes de crise econômica. Rev Saude Publica 2003; 37(6):699-706.

22. Soares ES, Menezes GMS. Fatores associados à mortalidade neonatal precoce: análise de situação no nível local. Epidemiol. Serv. Saúde 2010; 19(1):51-60.

23. Szwarcwald CL, Chequer P, Castilho EA. Tendências da mortalidade infantil no Brasil nos anos 80 . Informe Epidemiológico do SUS 1992; 1:35-50.

24. Costa MCN, Mota ELA, Paim JS, Silva LMV, Teixeira MG, Mendes CMC. Mortalidade infantil no Brasil em períodos recentes de crise econômica. Rev Saude Publica 2003; 37(6):699-706. 